ANDRZEJ STATECZNY, MARTA WLODARCZYK-SIELICKA, GRZEGORZ ZANIEWICZ

Maritime University of Szczecin

\title{
USING HIGH-DENSITY BATHYMETRIC DATA FOR THE PRODUCTION OF PRECISE ENC
}

\begin{abstract}
Due to an increasing trend of replacing traditional paper navigational charts with electronic ones, requirements for the creation of new ENC have been steadily growing. One example is the precise electronic navigational chart, which is based on accurate mapping of topographic and hydrographic elements. Information about depth of the waters contained on the charts are crucial for the safety of navigation. During the production of precise navigational charts, bathymetric information acquired by a multi-beam sonar system (or swath-bathymetry interferometric system) is used. A multi-beam sonar system emits several signal beams from a single transducer in different directions, which allows to cover a hundred percent of the survey area. The authors of the article carried out an analysis of bathymetric data in terms of differences in their preparation for the production of precise ENC.
\end{abstract}

Keywords:

bathymetry, ENC.

\section{INTRODUCTION}

Electronic navigational charts (ENC) are commonly used nowadays and allow for safe vessel maneuvering in harbor areas. ENC are produced according to the strict S-57 standard enforced by the International Hydrographic Organization (IHO) and visualized thanks to the Electronic Chart Display and Information System (ECDIS). S-57 (International Hydrographic Organization Transfer Standard for Digital Hydrographic Data) is a standard for digital hydrographic data exchange between hydrographic stations and data distribution to the sailors and other users. Due to the growing demand for water transportation, harbor authorities need to increase loading and unloading capacities, shortening vessel maintenance time and granting shore access to bigger and bigger vessels - the so called 'maximum units'. This is directly connected with the need to provide much more detailed electronic 
charts, showing terrain with great horizontal and vertical accuracy, as well as the current multi-density bathymetry and available in scales enabling the depiction of all objects necessary for vessel navigation, such as bollards and bumpers. Taking into account the precision requirements set for ENC map developers, the S-57 standard becomes insufficient for creating precise harbor charts. Furthermore, there is the incompatibility issue regarding accuracy with the S-44 (Standard for Hydrographic Surveys) data acquisition standard, set under the International Hydrographic Organization.

According to S-44, within the strictest bathymetric measurement class (special class), the horizontal accuracy should be $\pm 2 \mathrm{~m}$, while $\mathrm{S}-57$ sets it at $\pm 5 \mathrm{~m}$. In addition, the scales of harbor cells leave much to be desired of in terms of their accuracy level. Currently, charts of the most widely used harbors of the world, are produced in the following scales: 1:25000 for Antwerp, 1:15000 for Le Havre, 1:25000 for Rotterdam, 1:12500 for Oslo and 1:10000 for Singapore. For an accurate electronic harbor chart, the generally assumed scale is 1:1000 or smaller, with the accuracy of topographical objects equal to $+/-0,20 \mathrm{~m}$. Thus, it is safe to assume that the currently produced ENC maps, with their low level of detail and accuracy, take only the broadest navigational safety into consideration, which makes them inadequate for narrow passages or harbor maneuvering procedures.

Widespread computerization, increased demand for more advanced navigation safety enhancement systems, density of water traffic and merchandise transportation create the necessity to generate chart products as depicting the actual environment as closely as possible. Accurate high-density electronic harbor charts (PortENC) and bathymetric charts (BatyENC) created in concordance with the IHO S-57 and S-100 standards, are currently the fundament for safe navigation within harbor limits.

\section{PRECISE ENC CHARTS}

A navigational chart is the basic source of information for the navigator. Currently, analog charts are more and more often replaced by digital charts, that do a better job of providing safe navigation for the vessel. Using digital charts, a navigator can quickly access specific information relevant to them, assess the situation based on the constantly displayed position of the ship, as well as control and monitor the vessel's path. Standardized or non-standardized versions of electronic charts can be used for navigational purposes. The former constitute an element of a navigational information imaging system, while the latter are a part of the other Electronic Chart Systems (ECS) [10]. Depending on the purpose of a given electronic chart, their development is governed by strict production standards. One should specifically point out commercial charts, used by navigators in sea transport, governed by the IHO S- 57 
and the DigestC standards, the latter being the standard for developing charts for military purposes. Both standards govern two different products, that constitute electronic charts. The primary focus of the article is the S-57 standard.

The elements required to appear on an electronic navigational chart have been defined by the catalog of objects and their attributes as specified by ENC. An ENC map includes not only information to be found on traditional, conventional charts, but also data from the Navigational Lights and Signals Charter, Nautical Radiostation Charter, as well as partial pilotage information. The level of accuracy, precision and scale required for the production of ENC cells does not meet the requirements set by users of narrow passages and channels, as well as maneuvering between harbor piers, where depth is the main safety issue. For example, the Szczecin harbor chart was created in the scale of 1:10000, generating only a general representation of the harbor basin arrangement, creating a direct threat for the navigation of maximum sized vessels. Electronic charts created under the constraints of S-57 are fairly imprecise, due to the source data error margins appointed by the standard. Errors of individual objects accumulate to hamper the overall precision of the chart.

The constant demand for sea transport causes the need to constantly improve the quality of the produced navigational systems, thus also creating demand for electronic charts depicting high-density bathymetry. Precise electronic navigational charts must meet much higher standards in terms of the accuracy of the represented data and providing navigational safety than standard ENC charts. Such charts will be used by the navigator during precise maneuvering: mooring, turning, docking, etc. In order to provide the maximum level of safety, a precise chart must contain highly detailed bathymetric and topographical data. The quality, precision and timeliness of data, as well as the scale of the chart, are also of the essence.

The accuracy given by the S-57 standard is described as the zone of confidence. It is an element directly affecting the precision of the chart and generating the requirements governing data acquisition methods. Table 1 depicts the accuracies for the strictest category of A1 areas, demanding highly precise measurements, including full bottom sweeps and depth designation for detected objects.

Table 1. Chart accuracies for A1 [7]

\begin{tabular}{|l|l|l|l|}
\hline Zone of confidence & Position accuracy & \multicolumn{2}{|c|}{ Depth accuracy } \\
\hline \multirow{4}{*}{$\mathrm{A} 1$} & \multirow{4}{*}{ $\pm 5 \mathrm{~m}$} & $=0.50+1 \% \mathrm{~d}$ \\
\cline { 3 - 4 } & & Depth $(\mathrm{m})$ & Accuracy $(\mathrm{m})$ \\
\cline { 3 - 4 } & & 10 & \pm 0.6 \\
& & 30 & \pm 0.8 \\
& & 100 & \pm 1.5 \\
& & 1000 & \pm 10.5 \\
\hline
\end{tabular}

where: $\mathrm{d}$ - average depth within the given body of water 
The governing standard for accuracy of bathymetric data is the S- 44 , created to ensure safety of navigation. The latest, fifth, edition of said publication - was published in February 2008, replacing the fourth edition from 1998. The S-44 publication governs the basic methods of carrying out hydrographic measurements, as well as the classification of water bodies in terms of accuracy requirements for the hydrographic operations applied. It contains a detailed protocol for grading depth measurement accuracy and the positioning during the works, while also defining the allowed errors for individual areas. Recommendations are also outlined as to the manner in which probing measurements should be performed. The standard also includes outlines in regards to controlling measurement data, as well as their processing and storing in databases.

The S-44 standard comprises a set of minimal requirements, that should be met while conducting hydrographic operations, mainly for cartographic purposes and maintaining the safety of navigation. State hydrographic authorities should introduce the standard or its equivalent into their local procedures.

The S-44 classifies bathymetric measurements based on their accuracy into 4 categories, each with different accuracy requirements for positioning and bathymetry. In order to produce a more accurate chart, the authors based their work on the Special Category of the S-44 Standard, while at the same time attempting to maximize the accuracies included within. It is the strictest category of bathymetric measurements, applicable for areas with critical water reserves underneath the vessel's keel. Due to this, it is necessary to carry out a full sweep of the measured body of water in regards to navigational obstacles. Special category basins are usually: mooring stations, harbors and canals [6]. Table 2 presents the accuracies given by the S- 44 standard for said category.

Table 2. Measurement accuracies under the S-44 Special Category [6]

\begin{tabular}{|c|c|}
\hline Category & Special category S-44 \\
\hline Basin description & $\begin{array}{l}\text { Basins with critical water } \\
\text { supply under the keel }\end{array}$ \\
\hline Maximum acceptable horizontal error ${ }^{*}$ & $2 \mathrm{~m}$ \\
\hline Maximum acceptable vertical error ${ }^{*}$ & $\begin{array}{l}\mathrm{a}=0,25 \mathrm{~m} \\
\mathrm{~b}=0,0075\end{array}$ \\
\hline $\begin{array}{l}\text { Positions of navigational signalization measures } \\
\text { and navigationally valid topographical objects }\end{array}$ & $2 \mathrm{~m}$ \\
\hline $\begin{array}{l}\text { Positions of shore lines and less navigationally valid } \\
\text { topographical elements }\end{array}$ & $10 \mathrm{~m}$ \\
\hline Floating navigational signalization measures ${ }^{*}$ & $10 \mathrm{~m}$ \\
\hline
\end{tabular}

where: a - inaccuracy compound, irrespective of depth; $b$ - factor representing depth-dependant inaccuracy compound; $d$ - depth in meters; $b \times d-$ component of inaccuracy, that changes depending on depth. 
S-57 assumes a horizontal accuracy of $5 \mathrm{~m}$, while according to S-44 it should be $2 \mathrm{~m}$. Considering the purpose of an accurate navigational chart, these requirements are not satisfactory. Judging on the existing standard for creating electronic navigational charts, the authors opted to minimize accuracy errors of the chart while collecting topographical and bathymetric data.

Using the GPS/RTK positioning system (working at ASG-EUPOS), shore line positioning data was collected, along with other harbor-specific elements, such as: bumpers, bollards, navigational lighting, etc. All the data was acquired with an RMS error below $5 \mathrm{~cm}$. The bathymetry was collected using an interferometric bathymetric system, also positioned using the GPS/RTK system. The resulted accuracies range from 5 to $10 \mathrm{~cm}$.

Table 3. Comparison of requirements in accuracy [own study]

\begin{tabular}{|l|l|l|l|}
\hline & Standard S-44 & Standard S-57 & Authors of the article \\
\hline Positioning accuracy* & $2 \mathrm{~m}$ & $0.6 \mathrm{~m}$ & $0.05 \mathrm{~m}$ \\
\hline Depth accuracy* & $0.26 \mathrm{~m}$ & $0.51 \mathrm{~m}$ & $0.05 \mathrm{~m}$ \\
\hline
\end{tabular}

Analyzing the results, one can affirm that the gathered data is representative for creating accurate navigational charts. The accuracy of chart elements largely depends on the measurement system used, as well as the possessed data. From the point of time and economy, the chart requires a lengthy development process and a lot of manpower. Hence, these charts should be dedicated to harbor areas, where traffic density and vessel navigation involve a high level of risk - efficiently minimized with the use of accurate electronic charts.

\section{HIGH-DENSITY BATHYMETRIC DATA}

Contemporary bathymetric measurement systems allow to record more and more data of increasingly high quality. Multi-beam sonars and interferometric systems represent systems able to collect high density data. Authors of the hereby article used the interferometric sonar system GeoSwath Plus $250 \mathrm{kHz}$ for collecting bathymetric data.

The GeoSwath sonar uses a different technique of forming an acoustic beam than it is traditionally realized in multi-beam systems. The measurement is performed by means of a phasic comparison of the measured angle at which the beam is deflected off the bottom. Each of the two heads of the system is comprised of a single transmitting disc and multiple receiving discs of similarly shaped beams. The amplitude and phase of the signal dispersed off the bottom surface are measured by electronic 
circuitry (fig. 1). The relative phasic delay (time delay measurement) between the head's receiving discs is decoded as the angle of the acoustic signal's return. The measurement accuracy of the return signal's angle is measured to a fraction of a degree at very short time intervals, resulting in the high resolution of the data.

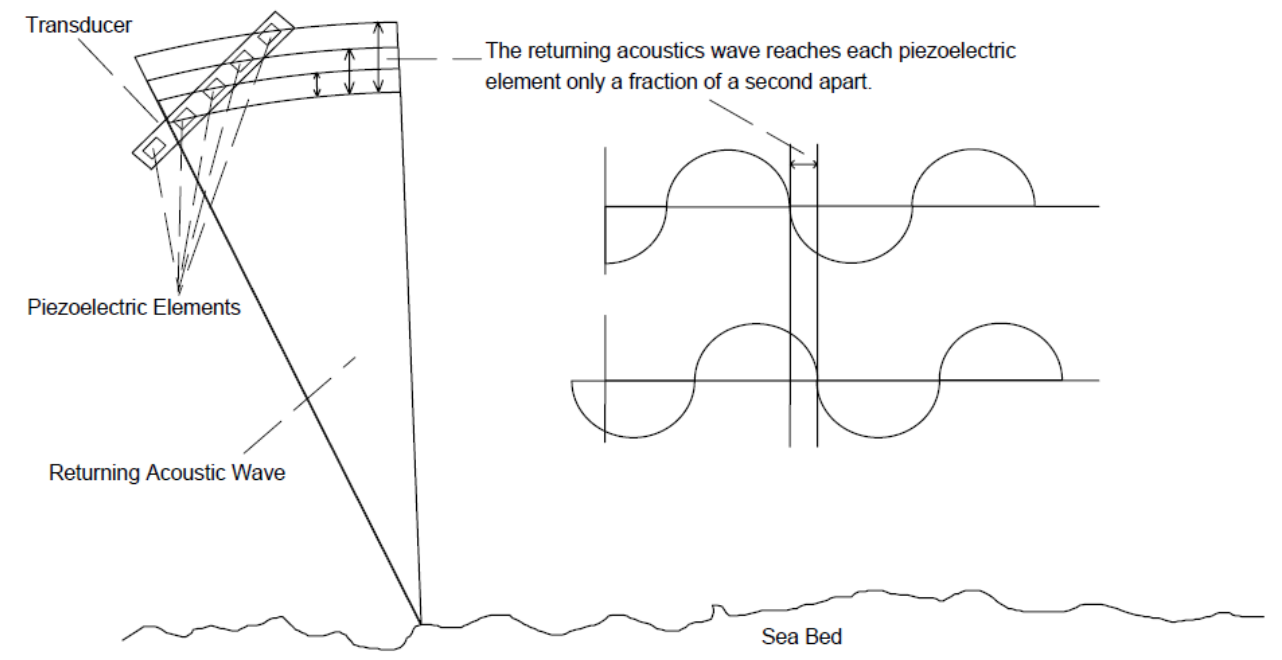

Fig. 1. Scheme and operating principle of the sonar head [1]

The beam formed out of transducers set at a $30^{\circ}$ angle has similar properties as the one generated in sonars - the angle width along the beam is determined above $150^{\circ}$, the width across - about $1^{\circ}$ [2]. In practice, this translates to a wide range of data coverage, estimated at about $7-8 \times$ depth.

The bathymetric measurements for future use in an accurate ENC product were based in the Szczecin Harbor area, near the Ewa elevator, which is a demonstrator of the Geo-technological System for Securing Operational Activities Involving Naval Defense of Harbors project realized by the Maritime University of Szczecin. A floating laboratory Hydrograf XXI, with the GeoSwath Plus $250 \mathrm{kHz}$ sonar and supplementary equipment (GPS/RTK, satellite compass, motion sensor) installed, was used to collect the data.

The measurement profiles were carried out so as to maintain a $100 \%$ coverage of the measured body of water.

\section{USING HIGH-DENSITY BATHYMETRIC DATA TO CREATE ENC}

In order to create the product of the accurate ENC map, software developed by the company Caris was used, including a data processing software package, enabling 
the processing of data from the point of acquisition to compiling the final ENC cell. The article focused on working with high density data collected from bathymetric measurements. The diagram below presents all the stages of creating an accurate ENC using depths collected with the interferometric system.

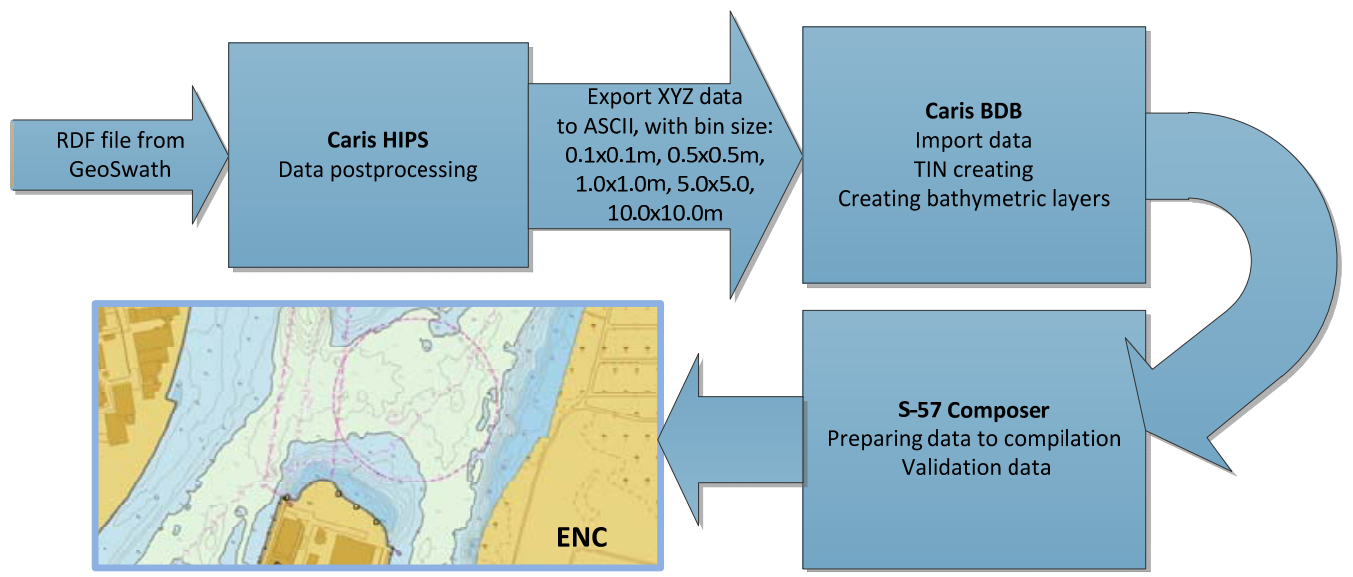

Fig. 2. Diagram of BathyENC development [own study]

In the initial phase, bathymetric data was processed in order to eliminate erroneous depths. The realization of this task was possible by implying robust filters from the HIPS package. The resulting swarm of points located in an XYZ spectrum was exported into ASCII files with the settings of $0.1 \times 0.1 \mathrm{~m}, 0.5 \times 0.5 \mathrm{~m}, 1.0 \times 1.0 \mathrm{~m}$, $5.0 \times 5.0 \mathrm{~m}, 10.0 \times 10.0 \mathrm{~m}$ and additionally saved as a raw data file. The authors chose the shallowest depth value, which is important for navigational safety. The statistical data from the exported values has been presented below.

Table 4. Statistics for the analyzed bin values [own study]

\begin{tabular}{|l|l|l|l|l|l|l|}
\hline & raw data & bin $0.1 \mathrm{~m}$ & bin $0.5 \mathrm{~m}$ & bin $1.0 \mathrm{~m}$ & bin $5.0 \mathrm{~m}$ & bin $10.0 \mathrm{~m}$ \\
\hline $\begin{array}{l}\text { Number } \\
\text { of values }\end{array}$ & 12343454 & 6926047 & 2176404 & 619564 & 26793 & 6999 \\
\hline Sum & 118761776.4 & 68679068.1 & 20261864.72 & 5651594.245 & 230464.445 & 57430.189 \\
\hline Minimum & 0.904 & 0.904 & 0.904 & 0.904 & 0.904 & 0.904 \\
\hline Maximum & 16.863 & 16.863 & 16.717 & 16.615 & 16.379 & 15.531 \\
\hline Mean & 9.62143792 & 9.916055739 & 9.30979024 & 9.121889337 & 8.601666293 & 8.205484926 \\
\hline $\begin{array}{l}\text { Standard } \\
\text { deviation }\end{array}$ & 1.570912651 & 1.445818915 & 1.744564521 & 1.857866203 & 2.189579828 & 2.384490692 \\
\hline
\end{tabular}




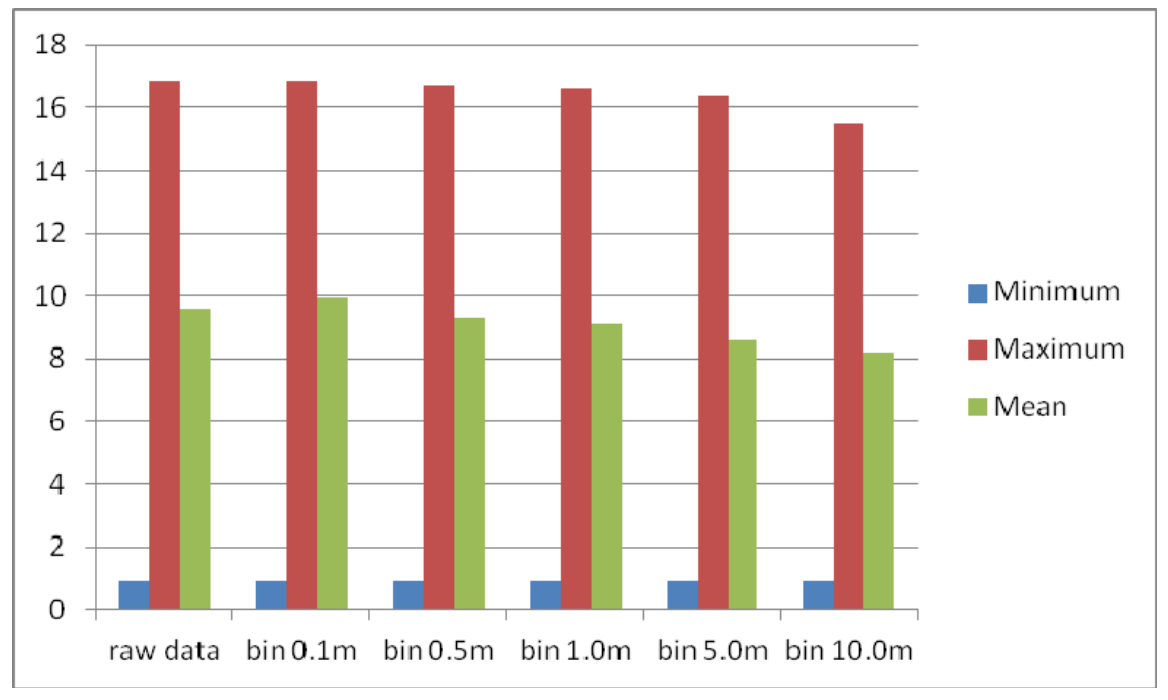

Fig. 3. Statistics for the analyzed bin values [own study]

Along with choosing a larger value for the bin size, the number of data decreases. The minimum depth value remains the same, which is consistent with hydrographic standards. This is crucial for navigational safety. The maximum depth value decreases with the increase of bin size value, so the average depth value also decreases with the increase of bin size value. The standard deviation dictates how widely the values are spread around its average. The lower the value of deviation, the more the observations focus around the average. The standard deviation value increases with the increase of bin size value.

In a subsequent step, ASCII files for individual bin values were imported into the BathyDataBase software, where the points were connected to one another with a net of irregular triangles (TIN), creating a surface for generating isobaths. For the purposes of developing an accurate ENC map, an interval of $1 \mathrm{~m}$ between the generated depth contours was adopted.

Additionally, the lines were smoothened using the B-spline algorithm and the lesser isobaths (not affecting safety) were filtered out, increasing the legibility of the chart. The generated depth areas for individual bin values were presented below (fig. 4).

The created bathymetric product was complemented with a layer representing the land area and the turntable. Due to adding the layer, one can deduce the visualization of data within the ECDIS system of a vessel traversing the area.

While analyzing the results in regards to creating an accurate navigational chart, one can point out the changes in the presentation of data occurring with the change in points used to generate the cloud. 
The arrangement of the generated isobaths and depth areas are best reflected in the measured bottom for the b. c. d. result. On account of an abundant degree of generalization in results e. f. one can point out the lacking accuracy, which is unacceptable when designing accurate ENC maps.
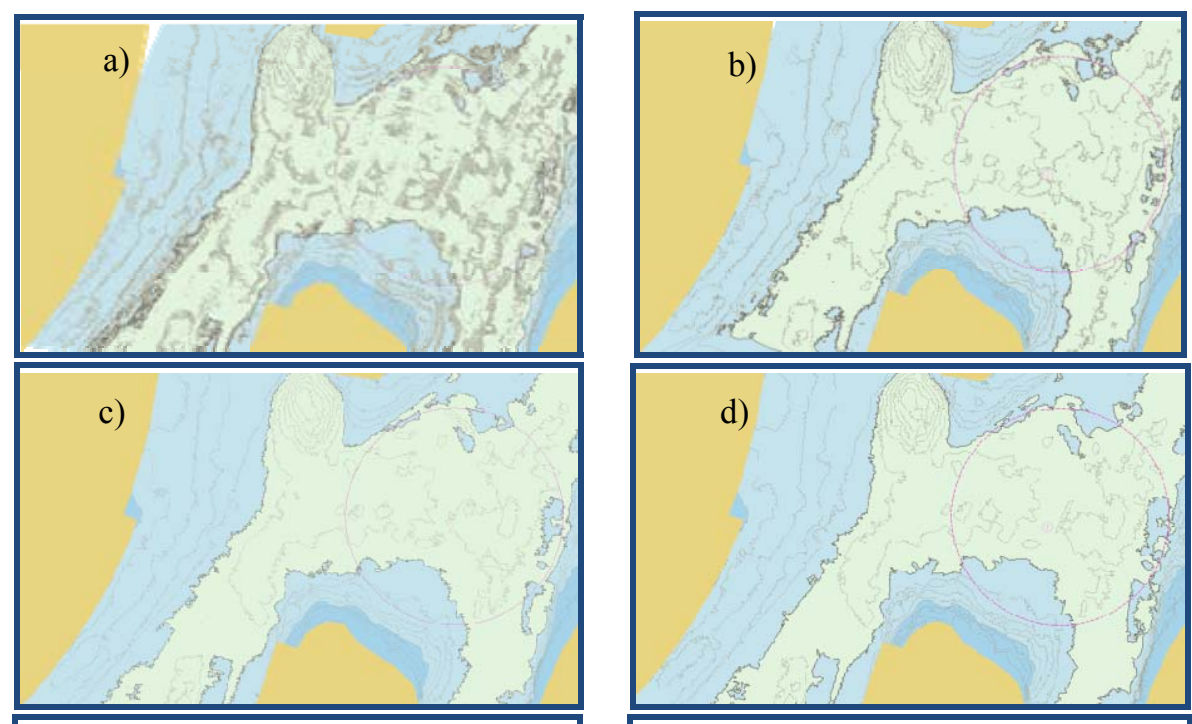

e)

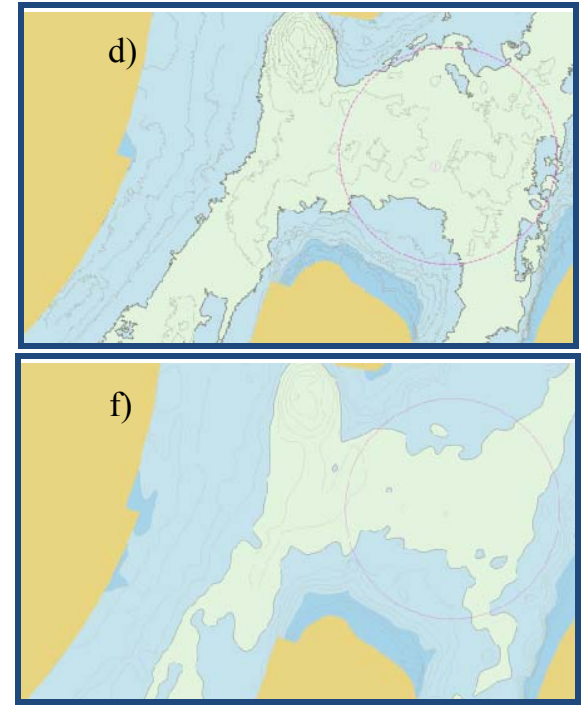

Fig. 4. Compiled results of the generated bathymetric layers with the resolution: a) raw data; b) 1 × $0.1 \mathrm{~m}$; c) $0.5 \times 0.5 \mathrm{~m}$; d) $1.0 \times 1.0 \mathrm{~m}$; e) $5.0 \times 5.0 \mathrm{~m}$; f) $10.0 \times 10.0 \mathrm{~m}$

The result obtained from the cloud of raw data points should not be used in the process of developing an accurate chart. Its depth areas are too 'ragged' and the peculiarity of raw data - accentuating each of the bottom features - leads to the creation of very small isobaths, which lessen the legibility of the result map.

\section{SUMMARY}

Electronic navigational charts allow for safe vessel navigation within harbor areas. Using them, the navigator can quickly access necessary information, assess 
the situation based on the constantly displayed vessel positioning, as well as control and monitor the course of the vessel. While creating an accurate ENC map, the authors adopted the S-44 Standard Special Category, while at the same time attempting to maximize the accuracies contained within. Due to the use of accurate navigational charts, harbors can accommodate larger and larger vessels. Choosing the greater bin size value for high density bathymetric data processing, the number of result data decreases. Setting an abundantly high cell value can lead to an excessive averaging of the results, thus arriving at inapplicable data. At the same time, using an overabundant quantity of data can lead to the navigational chart being illegible. Visualization and the proper generation of isobaths extensively affects the presentation of data on the given chart. The values chosen during the processing of data must each be thoroughly considered, having previously analyzed the expected results.

\section{REFERENCES}

[1] GeoAcustics, GeoSwath Plus Shallow Water Wide Swath Bathymetry System User Guide, 2005.

[2] Gerard Llort-Pujol et al., Advanced interferometric techniques for highresolution bathymetry, Journal Of Marine Technology Society, 2012.

[3] IHO, 1996. Specification for Chart Content and Display Aspects of ECDIS Ed.5.0.Special Publication No. 52.

[4] IHO, 2000. Transfer Standard for Digital Hydrographic Data, Ed.3.1.Special Publication No. 57.

[5] IHO, 2007. Annex A to Circular Letter 108/2007.IHB File No. S3/8151/CHRIS.

[6] IHO, 2008. Standards for Hydrographic Surveys, 5th Edition, February 2008. Special Publication No. 44.

[7] IHO, 2009. Transfer Standard For Digital Hydrographic Data. Supplementary Information for the Encoding of S-57 Edition 3.1 ENC Data (S-57 Supplement No. 2).

[8] Inland ENC Harmonization Group, 2008a. Inland Electronic Navigational Chart Encoding Guide, Edition 1, version 3.1.

[9] Inland ENC Harmonization Group, 2008b. Inland ENC Feature Catalogue, Edition 2.1.

[10] Łubczonek J., Włodarczyk M., The creation and the actualization of electronic maps for the inland navigation with the utilization of digital images by means of remote sensing, Archive of Photogrammetry, Cartography and Remote Sensing, 2009, Vol. 20.

Received May 2012

Reviewed October 2012 